\title{
Transbronchial needle aspiration of a mediastinal mass: therapeutic implications
}

\author{
KO PEN WANG, STEVE NELSON, JOHN SCATARIGE, STANLEY SIEGELMAN \\ From the Departments of Medicine and Radiology, Johns Hopkins University School of Medicine, Baltimore, \\ Maryland, USA
}

Neoplasms, cysts, inflammatory processes, and aneurysms may present as a mediastinal mass. New diagnostic techniques have recently become available for use in the investigation of a mediastinal mass, and we present a case illustrating the use of a transbronchial needle through a flexible bronchoscope to drain an accumulation of mediastinal fluid. So far as we are aware this is the first such report published in English.

\section{Case report}

A 71-year-old white man with a one-week history of cough was seen by a local practitioner. A chest radiograph showed a right paratracheal mass (fig 1a). A chest radiograph obtained three months previously was normal. The patient was admitted to hospital for evaluation.

The patient denied chest pain, dyspnoea, fever, night sweats, sputum production, and haemoptysis. He had a 35 pack-year history of cigarette smoking and had stopped smoking in 1963. He had had no known exposure to industrial or environmental agents. There was no known previous exposure to tuberculosis. He had had a malignant prostatic nodule treated by irradiation in 1977 . He had been treated for bladder malignancy in 1977 and 1981 by fulguration. His last cystoscopy, three months before admission, showed nothing abnormal. In 1979 a demand transvenous pacemaker had been inserted to control syncope.

Physical examination showed a well-developed, wellnourished white man with normal pulse, blood pressure, and temperature. There was no lymphadenopathy. All the findings on physical examination were entirely normal.

Laboratory investigation showed a packed cell volume of $38.1 \%$. Platelet and white blood cell counts were normal. Blood urea, plasma electrolyte, serum alkaline phosphatase, and acid phosphatase levels; urine analysis; and an electrocardiogram were all normal. The response to a tuberculin test was negative.

Computed tomography of the chest showed a right paratracheal mass $6 \mathrm{~cm}$ in diameter, which displaced the superior vena cava anteriorly and encroached on the right lateral tracheal wall. The appearance was consistent with carcinoma.

The patient underwent fibreoptic bronchoscopy and external compression of the right tracheal wall was noted with downward displacement of the right upper-lobe bron-

Address for reprint requests: Dr Ko Pen Wang, Brady 422 Respiratory Division, Johns Hopkins Hospital, Baltimore, Maryland 21205, USA.

Accepted 19 November 1982 chus. No endobronchial lesion was noted. Transbronchial aspiration of the right paratracheal area, with a 21-gauge type I transbronchial needle (Mill-Rose Company, Mentor, Ohio), was performed at the level of the azygos node. Sixty millilitres of serosanguineous fluid were aspirated. The fluid was consistent with an inflammatory exudate, histiocytes, inflammatory cells, and cellular débris being noted. Cytological examination gave negative results. Gram staining showed no organisms and no acid-fast bacilli were found. Bacterial and fungal cultures were negative.

Oesophagoscopy showed no evidence of mucosal abnormality and biopsy specimens were negative for malignancy. The mediastinal collection was thought to be most consistent with a sterile abscess. For therapeutic reasons a repeat right paratracheal needle aspiration was performed and an additional $80 \mathrm{ml}$ of fluid was obtained. Cytological examination and cultures again gave negative results. A repeat chest radiograph one day after the second aspiration showed appreciable reduction in the fluid collection (fig $1 b$ ).

The patient was discharged home and subsequent chest radiographs and a computed tomography scan after five months show no recurrence of the mediastinal abnormality.

\section{Discussion}

Abnormalities in the paratracheal and carinal areas are traditionally managed by surgical means, including mediastinoscopy, parasternal exploration, and thoracotomy. As neoplastic involvement of these areas generally precludes curative resection, transbronchial needle aspiration has been proposed as a safe and efficacious method of diagnosing and staging lung cancer. ${ }^{1}$

Transbronchial needle aspiration via a rigid bronchoscope has been shown to be a sensitive and specific alternative to more invasive surgical techniques used in the diagnosis of malignancies with mediastinal involvement. ${ }^{2}$ Similar results were obtained in a series of 15 patients with the flexible bronchoscope and transbronchial needle aspiration. $^{3}$

This procedure is useful in sampling the paratracheal, subcarinal, and hilar regions. In this patient a right paratracheal needle aspiration was performed by inserting the needle in the second and third intercartilaginous spaces, the carina being used as a landmark. Figure 2 shows the technique in use in another patient undergoing diagnostic aspiration of a right paratracheal mass.

In summary, this case shows that transbronchial needle 

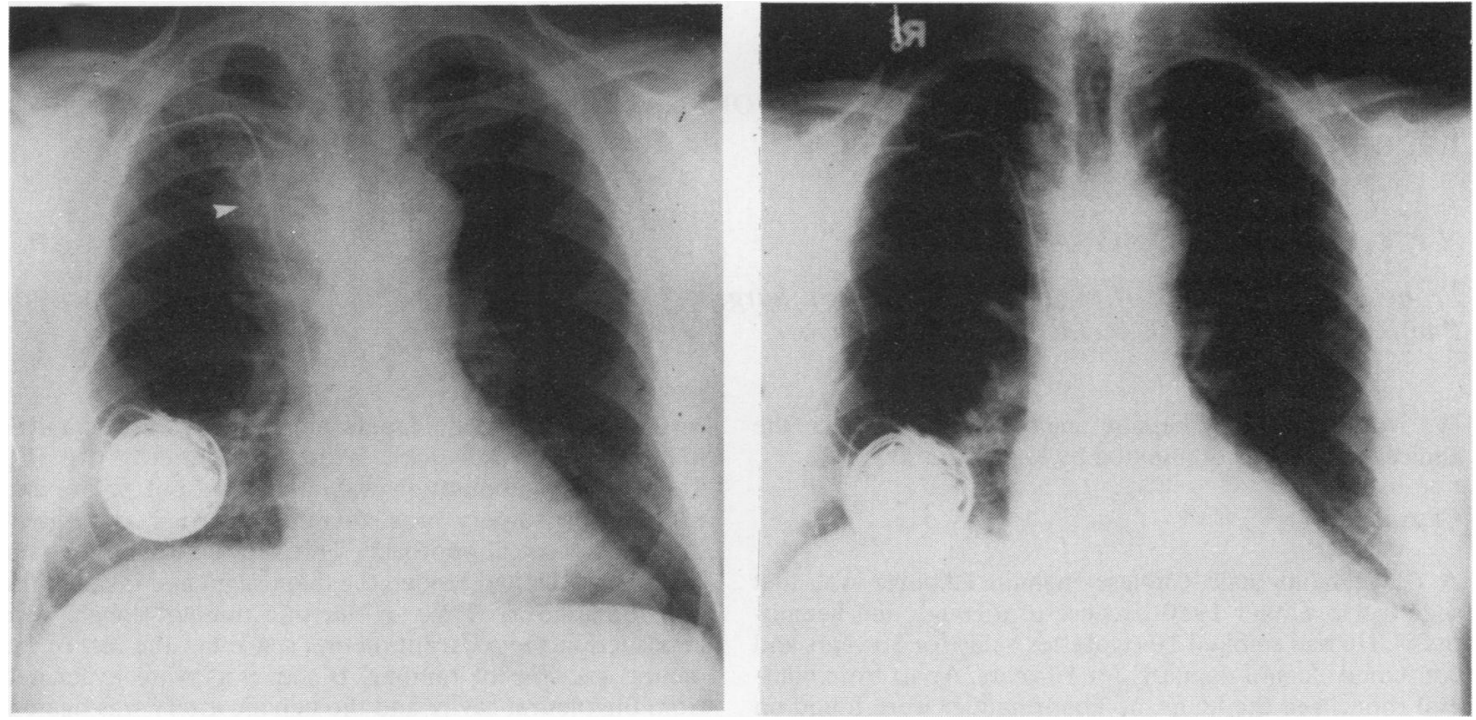

Fig 1 The patient's chest radiographs, (a) showing right paratracheal mass (arrow) and (b) after transbronchial needle aspiration.

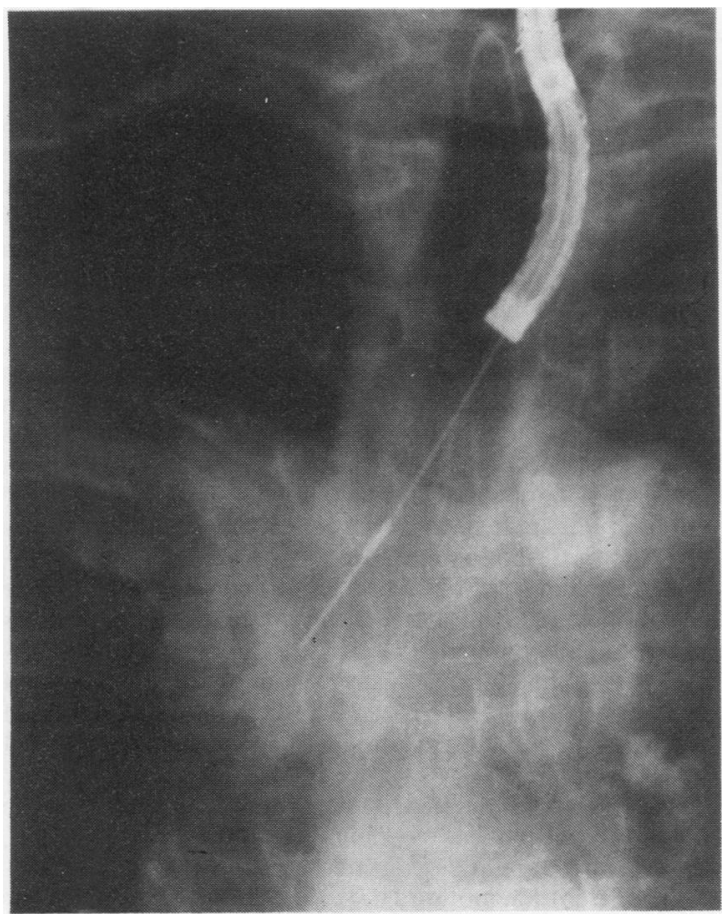

aspiration via fibreoptic bronchoscopy not only provides an accurate diagnosis in an expeditious and safe manner but also has therapeutic potential.

The authors wish to thank Dr William Schlott for referring this patient for bronchoscopy.

\section{References}

' Wang KP, Terry PB, Marsh BR. Bronchoscopic needle aspiration biopsy of paratracheal tumors. Am Rev Respir Dis 1978;118:17-21.

2 Wang KP, Marsh BR, Summer WR. Transbronchial needle aspiration for diagnosis of lung cancer. Chest 1981;80:48-50.

${ }^{3}$ Wang KP, Terry PB. Transbronchial needle aspiration in the diagnosis and staging of lung cancer. Chest 1981;80:342-3.

Fig 2 Transbronchial needle aspiration of a right paratracheal mass in another patient. 